\title{
Underestimation of COVID-19 mortality during the pandemic
}

\author{
Stacey Kung (101, Marjan Doppen", Melissa Black (10), Irene Braithwaite (101, \\ Ciléin Kearns ${ }^{1}$, Mark Weatherall ${ }^{2}$, Richard Beasley (i] ${ }^{1,3}$ and Nethmi Kearns ${ }^{1,3}$
}

Affiliations: ${ }^{1}$ Medical Research Institute of New Zealand, Wellington, New Zealand. ${ }^{2}$ Dept of Medicine, University of Otago, Wellington, New Zealand. ${ }^{3}$ Capital and Coast District Health Board, Wellington, New Zealand.

Correspondence: Stacey Kung, Medical Research Institute of New Zealand, Private Bag 7902, Wellington 6242, New Zealand. E-mail: stacey.kunglamrinz.ac.nz

\section{ABSTRACT}

Background: There has been considerable international variation in mortality during the COVID-19 pandemic. The objective of this study was to investigate the differences between mortality registered as due to COVID-19 and the excess all-cause mortality reported in countries worldwide during the COVID-19 pandemic.

Methods: Ecological analysis of 22 countries compared 5-year historical all-cause mortality, reported allcause mortality and expected all-cause mortality (calculated as historical mortality plus the reported deaths attributed to COVID-19). Data available from the first week of January 2020 to that most recently available were analysed.

Results: Compared to the preceding 5 years, there was an excess of 716616 deaths, of which $64.3 \%$ were attributed to COVID-19. The proportion of deaths registered as COVID-19-related/excess deaths varied markedly between countries, ranging between $30 \%$ and $197 \%$ in those countries that had an excess of deaths during the period of observation. In most countries where a definite peak in COVID-19-related deaths occurred, the increase in reported all-cause mortality preceded the increase in COVID-19 reported mortality. During the latter period of observation, a few countries reported fewer all-cause deaths than the historical figures.

Conclusion: The increases in all-cause mortality preceded the increase in COVID-19 mortality in most countries that had definite spikes in COVID-19 mortality. The number of deaths attributed to COVID-19 was underestimated by at least 35\%. Together these findings suggest that calculation of excess all-cause mortality is a better predictor of COVID-19 mortality than the reported rates, in those countries experiencing definite increases in mortality.

@ERSpublications

In countries experiencing marked increases in all-cause mortality during the global pandemic, all-cause mortality more accurately estimates \#COVID19 mortality and the onset of the pandemic than the reported COVID-19 mortality rates https://bit.ly/3fOtBJg

Cite this article as: Kung S, Doppen M, Black M, et al. Underestimation of COVID-19 mortality during the pandemic. ERJ Open Res 2021; 7: 00766-2020 [https://doi.org/10.1183/23120541.007662020].

This article has supplementary material available from openres.ersjournals.com

Data availability: The data analysed in this study were sourced from publicly available datasets provided by the Financial Times and Statistics New Zealand and have been appropriately referenced in the text.

Received: 20 Oct 2020 | Accepted: 25 Nov 2020

Copyright $\odot$ ERS 2021. This article is open access and distributed under the terms of the Creative Commons Attribution Non-Commercial Licence 4.0. 


\section{Introduction}

Since December 2019 and as of November 22, 2020, there have been over 58 million reported cases of COVID-19 and over 1.38 million deaths registered as due to COVID-19 [1]. The consequences of the pandemic in countries across the globe have been disparate and at times of marked contrast. There has been considerable international variation in mortality during the COVID-19 pandemic, which may both reflect and be used to guide policy and public health measures [2-6]

Two reports based on US data are consistent with underestimation of mortality due to COVID-19 during the first weeks of the pandemic [7,8]. Deaths attributed to COVID-19 only accounted for two-thirds of the estimated excess deaths during this period, with likely over-reporting of mortality due to other nonrespiratory illnesses. The five states with the most COVID-19 deaths experienced large proportional increases in deaths registered as due to diabetes, heart disease, Alzheimer's disease and cerebrovascular diseases.

An analysis of international excess all-cause mortality figures using data from the Human Mortality Database and the European Centre for Disease Prevention and Control has also suggested that there have been misclassifications of the causes of excess mortality [9]. However, for the 10 countries included in this analysis, data were only included from the week of the first reported COVID-19 death [9]. Considering that there was limited testing in the early stages of the COVID-19 breakout, a more comprehensive understanding can be obtained from analysis of excess mortality from the beginning of 2020 through to currently available mortality data.

In this study, we report an analysis of mortality from 22 countries, exploring patterns and time trends of mortality associated with the COVID-19 pandemic from the first week of January 2020. The objective of this study was to investigate the differences between the mortality registered as due to COVID-19 and the excess all-cause mortality reported in countries worldwide during the COVID-19 pandemic. Our hypothesis was that there would be marked underestimation of mortality registered as due to COVID-19 internationally, when calculated as a proportion of the excess deaths.

\section{Methods}

Reported weekly all-cause and historical weekly all-cause mortality, based on date of death, were obtained from the COVID-19-related data repository from the Financial Times [10]. These data were available for 21 countries that met the following criteria: (1) reported daily or weekly all-cause mortality figures during 2020; and (2) reported at least one full year of historical mortality figures, but preferably had data available for the 2015-2019 period. Data from all 21 countries were included in our analysis.

Mortality data for New Zealand were not available from the Financial Times and therefore was obtained from Stats NZ Tatauranga Aotearoa [11], the New Zealand official data agency. The Stats NZ dataset reported weekly number of deaths in New Zealand from 2015 to 2019; these figures are based on the date of death. As deaths in New Zealand are often registered later than the date of death, these data are subject to revisions over time. The figures reported in this study reflect the data available as at October 14, 2020.

Daily mortality registered as COVID-19-related was obtained from Our World in Data for all 22 countries [12]. These COVID-19-related mortality figures reflect the date that the deaths were reported. The weekly COVID-19 deaths for each country were calculated for the corresponding weeks reported in the Financial Times and Stats NZ datasets. Data available from as early as the first week of January 2020 to that most recently available (at the time of submission) were analysed.

The Financial Times data repository reported historical mortality as the median number of deaths per week for 2015 to 2019. To ensure the reporting of historical mortality figures for New Zealand was consistent with the Financial Times data, the median number of deaths per week for 2015 to 2019 in New Zealand was calculated using the Stats NZ dataset. Reported all-cause mortality was the number of reported deaths per week in 2020 for all 22 countries, which was extracted from the Financial Times and Stats NZ datasets. The difference between reported deaths and historical deaths per week was calculated to estimate the excess deaths during this period. The expected mortality for the corresponding weeks in 2020 were calculated as the historical mortality plus the reported deaths that were attributed to COVID-19. The ratio of deaths registered as COVID-19-related/excess deaths was calculated to estimate the proportion of excess deaths that were attributed to COVID-19.

\section{Results}

The time course of reported all-cause mortality, historical all-cause mortality and expected all-cause mortality for the European countries and non-European countries are shown in figure 1a and b, respectively. The individual figure for each country is shown in the supplementary material. There were 6162160 deaths (reported all-cause mortality) in the 22 countries analysed over the reported period (table 1). Compared to the preceding 5 years, this was an excess of 716616 deaths, of which $64.3 \%$ were attributed to COVID-19. 
a)
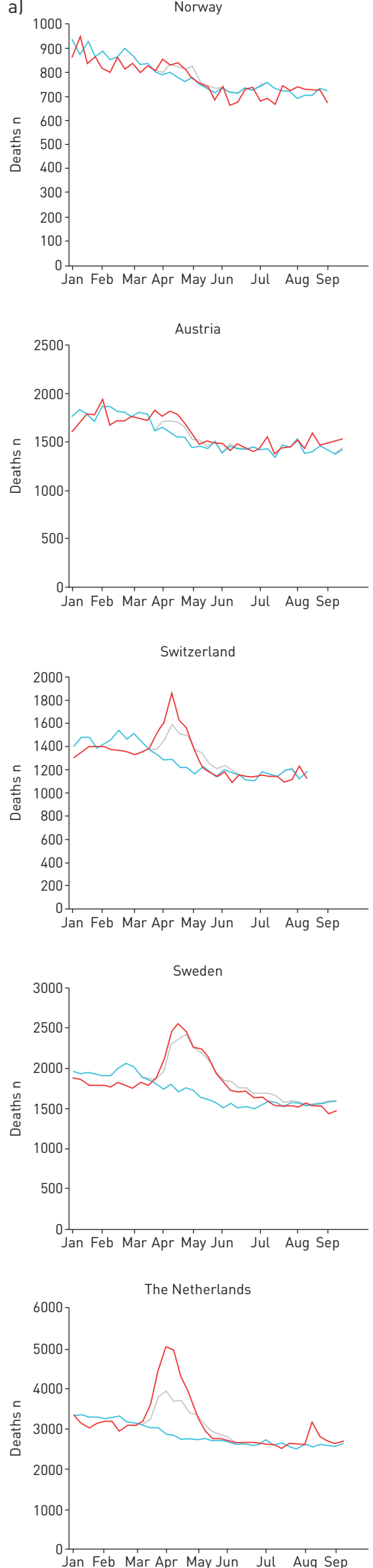
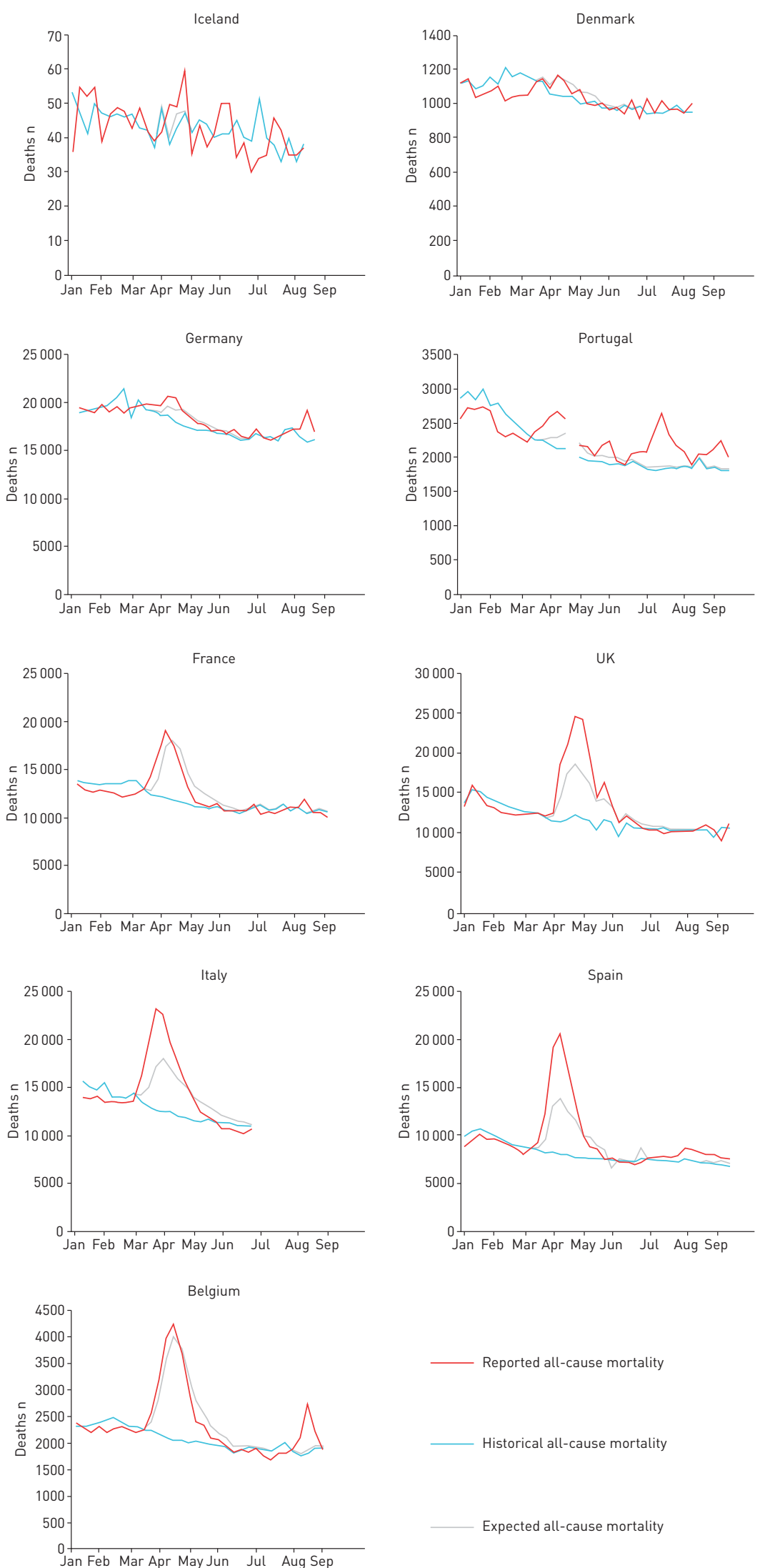

FIGURE 1 The weekly reported all-cause mortality in 2020, historical all-cause mortality based on previous 5-year mortality data and expected all-cause mortality calculated from historical all-cause mortality plus COVID-19 mortality data in a) 14 European countries (the Financial Times had 1 week of data missing in April for Portugall and b) eight non-European countries. 
b)
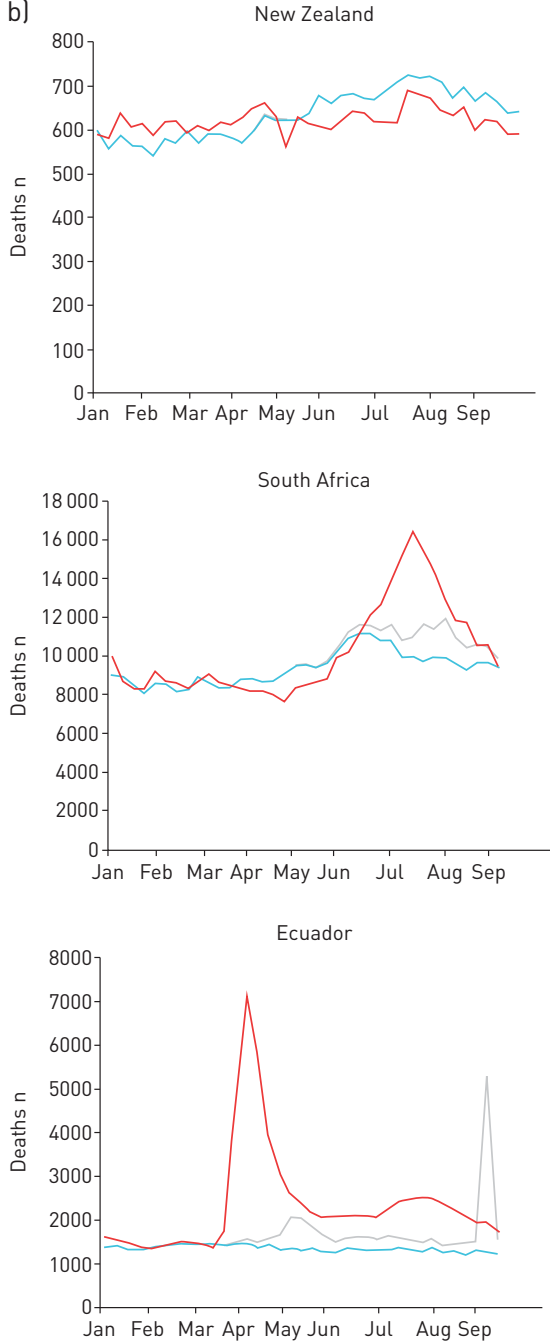
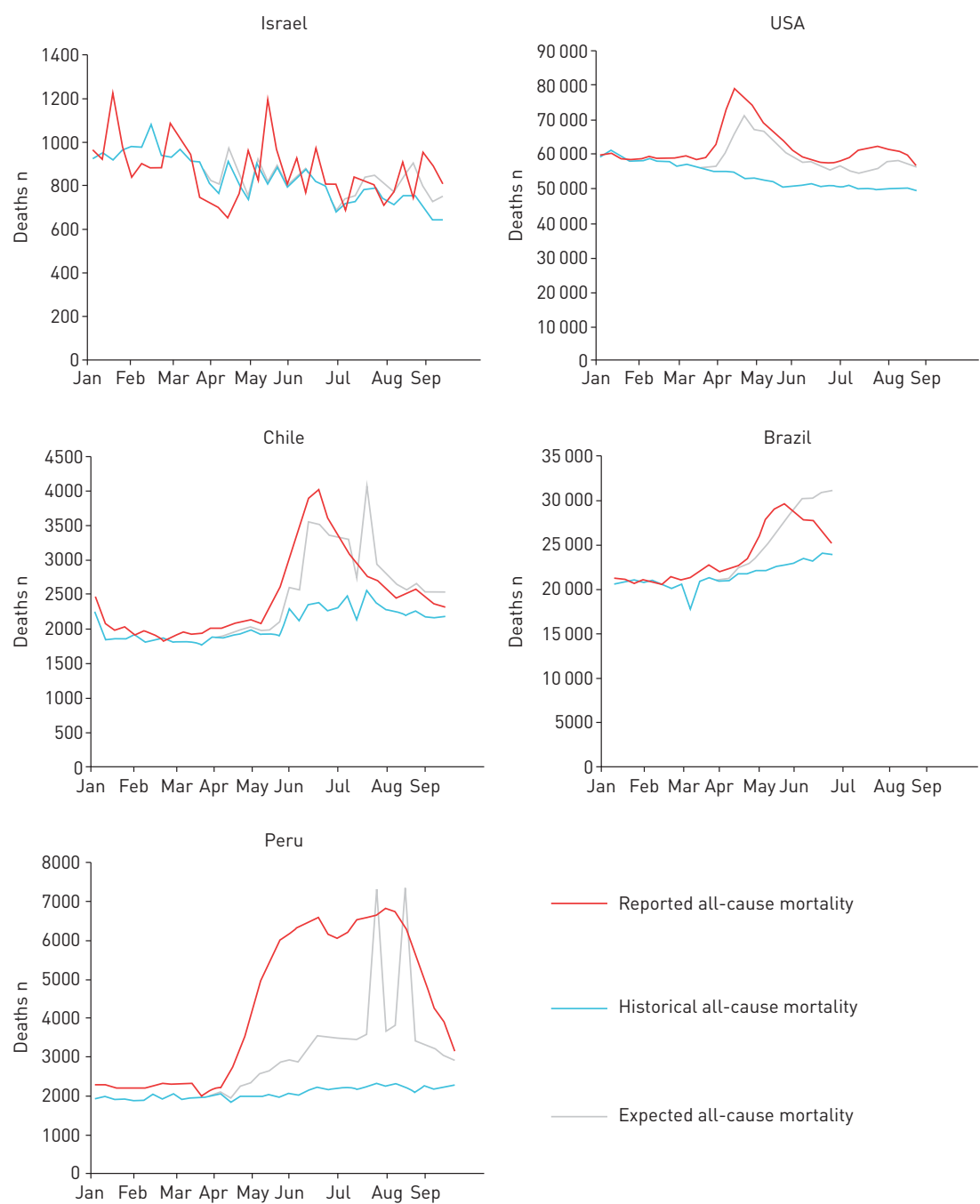

Reported all-cause mortality

_ Historical all-cause mortality

Expected all-cause mortality

FIGURE 1 Continued.

There was marked variation between countries in the proportion of excess deaths that could be attributed to reported cases of COVID-19, ranging between $30 \%$ and $197 \%$ in those countries that had an excess of deaths during the period of observation.

There was a marked variation in all-cause mortality, when compared with historical all-cause mortality, both within European and non-European countries. A number of countries had more than double the historical weekly all-cause mortality during the peak of the pandemic during this period, including Belgium (207\%), Ecuador (497\%), Peru (304\%), Spain (257\%) and the United Kingdom (201\%). Countries that had more than between 50 to $100 \%$ of the historical weekly all-cause mortality during the peak of the pandemic included Chile (170\%), France (159\%), Italy (185\%), the Netherlands (175\%), South Africa (165\%) and Sweden (150\%). Denmark, New Zealand and Norway had fewer deaths in 2020 compared to the previous years.

In most countries with definite increases in deaths attributed to COVID-19, the increase in all-cause mortality from the historical trends preceded the mortality registered as due to COVID-19. In most countries, the delay was around 2 weeks; however, in a few countries such as Sweden, Germany, Spain, the Netherlands and the United Kingdom there was no appreciable delay, whereas in Ecuador there was a delay of more than 4 weeks. This temporal pattern was also observed at the onset of the second major wave later in the pandemic in a number of countries including Belgium, Germany and the Netherlands.

During the latter period of the observation period, there were a few countries in which the number of deaths from all causes were less than the number predicted from the previous 5-year period. These included countries such as New Zealand and Norway, which did not experience clear peaks in COVID-19-related mortality during this period, and Italy which did. 
TABLE 1 Historical and reported all-cause mortality, excess deaths, reported COVID-19 deaths and proportion of excess deaths attributed to COVID-19, in 22 countries during the COVID-19 pandemic

\begin{tabular}{|c|c|c|c|c|c|c|}
\hline \multirow[t]{2}{*}{ Country } & \multirow{2}{*}{$\begin{array}{c}\text { Historical all-cause } \\
\text { deaths }{ }^{\#}\end{array}$} & \multirow{2}{*}{$\begin{array}{l}\text { Reported all-cause } \\
\text { deaths in } \\
2020\end{array}$} & \multirow{2}{*}{$\begin{array}{l}\text { Excess } \\
\text { deaths }\end{array}$} & \multicolumn{2}{|c|}{ COVID-19 deaths } & \multirow[t]{2}{*}{ Reported period } \\
\hline & & & & $\begin{array}{l}\text { Reported } \\
\text { deaths }\end{array}$ & $\begin{array}{c}\text { Proportion of excess } \\
\text { deaths }{ }^{ף 1} \%\end{array}$ & \\
\hline Belgium & 47913 & 56074 & 8161 & 9472 & 116.1 & 06 Jan-30 Aug \\
\hline Brazil & 518306 & 573822 & 55516 & 48954 & 88.2 & 04 Jan-19 Jun \\
\hline Chile & 76684 & 91690 & 15006 & 12058 & 80.4 & 02 Jan-16 Sep \\
\hline France & 411181 & 428554 & 17373 & 30698 & 176.7 & 06 Jan-06 Sep \\
\hline Germany & 587931 & 602115 & 14184 & 8668 & 61.1 & 06 Jan-23 Aug \\
\hline Iceland & 1372 & 1379 & 7 & 10 & 142.9 & 30 Dec-09 Aug \\
\hline Israel & 30817 & 32278 & 1461 & 1147 & 78.5 & 02 Jan-16 Sep \\
\hline Italy & 307680 & 347194 & 39514 & 27670 & 70 & 09 Jan-23 Jun \\
\hline Netherlands & 102945 & 113042 & 10097 & 6249 & 61.9 & 09 Jan-16 Sep \\
\hline South Africa & 346083 & 378059 & 31976 & 15499 & 48.5 & 01 Jan-15 Sep \\
\hline Spain & 298676 & 350594 & 51918 & 29848 & 57.5 & 30 Dec-13 Sep \\
\hline Sweden & 61605 & 65434 & 3829 & 5846 & 152.7 & 01 Jan-08 Sep \\
\hline Switzerland & 40988 & 41856 & 868 & 1711 & 197.1 & 30 Dec-09 Aug \\
\hline UK & 432109 & 491266 & 59157 & 41608 & 70.3 & $28 \mathrm{Dec}-11 \mathrm{Sep}$ \\
\hline USA & 1829331 & 2110542 & 281211 & 175406 & 62.4 & $29 \mathrm{Dec}-22 \mathrm{Aug}$ \\
\hline Total & 5445544 & 6162160 & 716616 & 460928 & 64.3 & \\
\hline
\end{tabular}

Data are presented as $n$ unless otherwise stated. * : calculated as the median number of deaths from the past 5 years (2015-2019) for the reported period; " : calculated as the number of reported COVID-19 deaths divided by the number of excess deaths and multiplied by 100.

\section{Discussion}

There are a number of international perspectives provided by this analysis of the mortality impact of COVID-19 and its reporting. Firstly, there is a marked variation in the trends in all-cause mortality between countries. Many countries reported a greater than $50 \%$ increase in mortality at the peak of the pandemic. However, a few countries that imposed early and stringent lockdown measures, reported none or a reduction.

Secondly, there was marked variation between countries in the proportion of excess deaths from historical rates that were registered as COVID-19-related, ranging between 30\% and 197\%, in those countries that had an excess of deaths during the period of observation. This variation is likely due to differences in healthcare systems, case definitions, availability of testing, variations in the historical baseline all-cause death rates from which the number of excess deaths were estimated, and other factors that contributed to deaths from causes other than COVID-19. As a result, country-specific COVID-19 mortality statistics should be interpreted and compared with caution. Both the variability and the magnitude of differences between countries, suggest that excess deaths, calculated as total all-cause mortality minus historical mortality for the same week from the previous 5 years, provides a better estimate of COVID-19 mortality than official reports of deaths registered as due to COVID-19, in those countries with definite increases in mortality. This interpretation does not apply to countries in which definite increases in all-cause mortality did not occur.

Potential explanations for the greater increase in all-cause mortality than reported COVID-19-related deaths include misclassification of deaths due to COVID-19 attributed to another cause, and deaths from causes indirectly related to COVID-19 such as cardiovascular [13] and thrombotic events [14]. Mortality from delayed seeking of medical care, avoidance of care, mental health distress, and exacerbation of chronic illnesses as a result of the response to the pandemic such as lockdowns may also possibly contribute to excess mortality. Furthermore, overwhelmed health systems and stretched capacity could potentially result in substandard management of non-COVID-19 related conditions, amplifying pre-existing deficiencies and inequities contributing to excess mortality. These effects could continue to 
contribute towards life-years lost and/or excess mortality for months and years to come. However, the full extent of the effects that the COVID-19 pandemic has had on health and mortality is currently unknown.

There are emerging data to support this interpretation of our findings. In England and Wales, there has been a general increase in mortality across the leading causes of death throughout the pandemic. Of note, from late March onwards there have been large increases in deaths attributed to dementia and Alzheimer's disease (220\% of 5-year average at maximum) and "symptoms, signs and ill-defined conditions" (242\% of 5-year average at maximum) [15]. The latter category is used to include deaths certified as caused by "old age" or "frailty". The majority of these deaths occurred in care homes where there was rapid infection and transmission in this vulnerable population. There was also an increase in deaths related to asthma and diabetes, which occurred at significantly higher rates compared to the preceding 5 -year average, potentially related to a delay in seeking or receiving treatment. Similarly, in the United States there were large proportional increases in deaths due to nonrespiratory causes such as diabetes, heart disease, cerebrovascular disease and Alzheimer's disease, likely in part due to misclassification of COVID-19-related deaths [7].

These findings are consistent with reporting of mortality in previous unexpected epidemics or catastrophic events. During the chikungunya epidemic in Jamaica, no chikungunya-associated deaths were reported despite an excess of 2499 deaths [16]. The mortality burden of 1139 excess deaths due to Hurricane Maria in Puerto Rico in 2017 was higher than the official death toll of 64 [17].

Thirdly, increases in all-cause mortality preceded the increase in COVID-19 mortality in most countries in which definite spikes in COVID-19 mortality occurred. This temporal pattern was also observed at the onset of the second major wave later in the pandemic. This further suggests that changes in all-cause mortality, compared with historical mortality rates, may be more informative than COVID-19-specific mortality in recognising the onset and extent of the early impact of COVID-19, in those countries in which definite increases in all-cause mortality occurred. This may also apply to other epidemics or pandemics due to a novel virus where there is a lack of knowledge and widespread testing or detection, and for which robust contract tracing may not be in place. Of note, Ecuador, which had the longest delay in reporting of COVID-19 mortality after the onset of the pandemic-related increase in all-cause mortality, had the greatest increase in mortality during the pandemic, recording a $497 \%$ increase at its peak. This observation suggests that the lack of recognition of the presence and extent of the pandemic may have contributed to the lack of adequate public health measures to contain the pandemic.

Finally, for a few countries with early and stringent lockdown measures, the reported all-cause mortality was less than historical rates at periods during the pandemic. Countries such as New Zealand, which did not experience a spike in COVID-19 mortality, may have had reduced mortality from other causes (e.g. motor vehicle accidents or other infectious respiratory causes such as influenza), due to stringent lockdown measures, and the general improvement in social and public health practices [18, 19]. In Italy, which experienced a marked spike in COVID-19 mortality, a late reduction in all-cause mortality may be due to premature death of older adults, including in residential care facilities. Thus, all-cause mortality may also provide insight into the net outcome of both the impact of COVID-19 and the public health measures implemented during the pandemic.

Limitations of this ecological analysis are that pandemic public health measures may have affected other causes of mortality and there are likely inaccuracies in country mortality databases, and in particular the registration of COVID-19-related deaths on which this analysis was based. The methodology used by countries in reporting COVID-19 mortality vary and some countries such as the United Kingdom, France and Spain have changed methods over the course of the pandemic [20]. Our World in Data also publishes the death on the date it is reported, whereas the Financial Times dataset uses national registry data, which are reported by date of death. This discrepancy may generate a potential lag depending on the efficiency of each country's systems but even when accounting for it, the difference in the time trends of reported all-cause mortality and reported COVID-19 mortality is evident. Using a 5-year average to calculate historical deaths does not account for changes in population over time. High-quality data can be difficult to obtain in a pandemic situation given the evolving nature of the disease and its burden on established reporting systems; however, the data used in this analysis are the most accurate that are currently available. Finally, there are no data from many other affected countries of interest such as China, Taiwan, South Korea and Australia.

We conclude that this analysis is consistent with the underestimation of COVID-19 mortality by at least one-third. We propose that all-cause mortality may be more informative than the COVID-19-specific mortality when gauging the impact of COVID-19 within countries, particularly early in the pandemic. The time trends reported suggest that the stringent public health measures to contain COVID-19, and the impact of the pandemic itself, may have led to a reduction in deaths from other causes, an observation that requires further investigation. 
Conflict of interest: None declared.

Support statement: No funding was sought or received for this study. The Medical Research Institute of New Zealand receives independent research organisation funding from the Health Research Council of New Zealand.

\section{References}

1 European Centre for Disease Prevention and Control. COVID-19 situation update worldwide, as of 22 November 2020. www.ecdc.europa.eu/en/geographical-distribution-2019-ncov-cases Date last accessed: November 23, 2020. Date last updated: 2020.

2 Nogueira PJ, De Araújo Nobre M, Nicola PJ, et al. Excess mortality estimation during the COVID-19 pandemic: preliminary data from Portugal. Acta Med Port 2020; 33: 376-383.

3 Pasquariello P, Stranges S. Excess mortality from COVID-19: a commentary on the Italian experience. Int J Public Health 2020; 65: 529-531.

4 Vestergaard LS, Nielsen J, Richter L, et al. Excess all-cause mortality during the COVID-19 pandemic in Europe preliminary pooled estimates from the EuroMOMO network, March to April 2020. Eurosurveillance 2020; 25: 2001214.

5 Pearce N, Lawlor DA, Brickley EB. Comparisons between countries are essential for the control of COVID-19. Int J Epidemiol 2020; 49: 1059-1062.

6 Nagpal R, He Y, Goyal N, et al. Comparison between India, USA and China on the basis of rigidity of public health measures with case count and mortality rate during COVID-19. Curr J Appl Sci Technol 2020; 39: 127-132.

7 Woolf SH, Chapman DA, Sabo RT, et al. Excess deaths from COVID-19 and other causes, March-April 2020 JAMA 2020; 324: 510-513.

8 Woolf SH, Chapman DA, Sabo RT, et al. Excess deaths from COVID-19 and other causes. JAMA 2020; 324 1562-1564.

9 Beaney T, Clarke JM, Jain V, et al. Excess mortality: the gold standard in measuring the impact of COVID-19 worldwide? J R Soc Med 2020; 113: 329-334.

10 Financial Times. Excess mortality during the COVID-19 pandemic. https://github.com/Financial-Times/ coronavirus-excess-mortality-data/tree/master/data Date last accessed: October 14, 2020. Date last updated: 2020.

11 Stats NZ Tatauranga Aotearoa. COVID-19 data portal. www.stats.govt.nz/experimental/covid-19-data-portal Date last accessed: October 14, 2020. Date last updated: 2020.

12 Roser M, Ritchie H, Oritz-Ospina E, et al. Coronavirus pandemic (COVID-19). https://ourworldindata.org/ coronavirus Date last accessed: October 14, 2020. Date last updated: 2020.

13 Guo T, Fan Y, Chen M, et al. Cardiovascular implications of fatal outcomes of patients with coronavirus disease 2019 (COVID-19). JAMA Cardiol 2020; 5: 811-818.

14 Bikdeli B, Madhavan MV, Jimenez D, et al. COVID-19 and thrombotic or thromboembolic disease: implications for prevention, antithrombotic therapy, and follow-up. J Am Coll Cardiol 2020; 75: 2950-2973.

15 Office for National Statistics. Analysis of death registrations not involving coronavirus (COVID-19), England and Wales: 28 December 2019 to 1 May 2020. Cardiff, ONS, 2020.

16 Freitas ARR, Gérardin P, Kassar L, et al. Excess deaths associated with the 2014 chikungunya epidemic in Jamaica. Pathog Glob Health 2019; 113: 27-31.

17 Santos-Lozada AR, Howard JT. Use of death counts from vital statistics to calculate excess deaths in Puerto Rico following Hurricane Maria. JAMA 2018; 320: 1491-1493.

18 Wilson N, Mizdark A, Summers J, et al. Weekly deaths declined in NZ's lockdown - but we still don't know exactly why. https://blogs.otago.ac.nz/pubhealthexpert/weekly-deaths-declined-in-nzs-lockdown-but-we-still-dontknow-exactly-why/. Date last accessed: August 28, 2020.

19 Baker MG, Wilson N, Anglemyer A. Successful elimination of Covid-19 transmission in New Zealand. N Engl J Med 2020; 383: e56.

20 Financial Times. Coronavirus tracked: see how your country compares. https://ig.ft.com/coronavirus-chart/? areas $=$ eur\&areas $=$ usa\&areas $=$ bra\&areas $=$ gbr\&areasRegional=usny\&areasRegional $=$ usca\&areasRegional=usfl\&areas Regional $=$ ustx \&byDate $=0 \&$ cumulative $=0 \& \operatorname{logScale}=1$ \&perMillion $=0 \&$ values $=$ deaths Date last accessed: August 28, 2020. Date last updated: 2020 